\title{
Computing the Volume, Counting Integral Points, and Exponential Sums*
}

\author{
Alexander I. Barvinok $\dagger$ \\ I. M. Sechenov Institute of Evolutionary Physiology and Biochemistry, \\ Academy of Sciences of Russia, 44 M. Thorez Prospect, \\ 194223 St. Petersburg, Russia \\ bar@ief.spb.su
}

\begin{abstract}
We design polynomial-time algorithms for some particular cases of the volume computation problem and the integral points counting problem for convex polytopes. The basic idea is a reduction to the computation of certain exponential sums and integrals. We give elementary proofs of some known identities between these sums and integrals and prove some new identities.
\end{abstract}

\section{Introduction}

Let $P \subset \mathbb{R}^{n}$ be a convex $n$-dimensional polytope. We consider the problem of computation of the volume of $P$ and counting the integral points in $P$. To do this we introduce the following main objects.

(1.1) Exponential Integral. We consider the following expression:

$$
\int_{P} \exp \{\langle c, x\rangle\} d x,
$$

where $c \in \mathbb{R}^{n},\langle\cdot$,$\rangle is the scalar product in \mathbb{R}^{n}$, and $d x$ is the Lebesgue measure on $\mathbb{R}^{n}$.

(1.2) Exponential Sum. We consider the following discrete sum:

$$
\sum_{x \in P \cap \mathbb{E}^{n}} \exp \{\langle c, x\rangle\}
$$

where $\mathbb{Z}^{n}$ is the standard integral lattice in $\mathbb{R}^{n}$.

* This research was partially supported by the Mittag-Leffler Institute.

† Current address: Department of Mathematics, Royal Institute of Technology, S-10044, Stockholm, Sweden. barvinok@math.kth.se. 
We also consider integrals and sums over a convex polytopal cone $K \subset \mathbb{R}^{n}$ :

$$
\int_{K} \exp \{\langle c, x\rangle\} d x \text { and } \sum_{x \in K \cap \mathbb{Z}^{n}} \exp \{\langle c, x\rangle\} \text {. }
$$

Exponential sums and integrals satisfy certain nontrivial algebraic relations which make it possible to compute them efficiently in some particular cases. Although the identities usually degenerate when $c=0$, the computation at a point $c$ close to 0 enables polynomial-time algorithms for computation of the volume and counting the integral points for some classes of polytopes to be designed.

In Section 2 we prove some identities for the exponential integrals. Then we use them to design algorithms for computation of the volume (Section 3). In Section 4 we prove certain identities for the exponential sums. Then we apply these identities for counting the integral points in integral polytopes (Section 5). In Section 6 we prove an anologue of identities for nonrational polytopes.

\section{Exponential Integrals}

We begin with three examples of exponential integrals over polytopes and cones.

(2.1) Example (Simple Cone). Let $K=\mathbb{R}_{+}^{n}$ be the nonnegative orthant, $\mathbb{R}_{+}^{n}=$ $\left\{x=\left(x_{1}, \ldots, x_{n}\right) \in \mathbb{R}^{n}: x_{i} \geq 0\right.$ for all $\left.i\right\}$. Choose $c \in \operatorname{Int} \mathbb{R}_{-}^{n}$, where $\mathbb{R}_{-}^{n}:=-\mathbb{R}_{+}^{n}$. Then we have

$$
\int_{K} \exp \{\langle c, x\rangle\} d x=\prod_{i=1}^{n}\left(-c_{i}^{-1}\right) .
$$

Moreover, let $K$ be the conic hull of linearly independent vectors $u_{1}, \ldots, u_{n}$, so $K=\operatorname{co}\left\{u_{1}, \ldots, u_{n}\right\}$. Denote by $K^{*}=\left\{x \in \mathbb{R}^{n}:\langle x, y\rangle \leq 0\right.$ for all $\left.y \in K\right\}$ the polar of $K$. Then for all $c \in \operatorname{Int} K^{*}$ we have

$$
\int_{K} \exp \{\langle c, x\rangle\} d x=\left|u_{1} \wedge \cdots \wedge u_{n}\right| \prod_{i=1}^{n}\left\langle-c, u_{i}\right\rangle^{-1} .
$$

To obtain the last formula we have to apply a suitable linear transformation to the previous integral.

(2.2) Example (Cube). Let $I^{n}=[0,1]^{n}$ be an $n$-dimensional unit cube. Then

$$
\int_{I^{n}} \exp \{\langle c, x\rangle\} d x=\prod_{i=1}^{n} c_{i}^{-1}\left(\exp \left\{c_{i}\right\}-1\right)
$$

for all $c=\left(c_{1}, \ldots, c_{n}\right)$. If $c_{i}=0$, then the corresponding factor is equal to 1 . 
(2.3) Example (Simplex). Let $L=\left\{x: x=\left(x_{1}, \ldots, x_{n}\right), x_{1}+\cdots+x_{n}=1\right\}$ be the hyperplane in $\mathbb{R}^{n}$ and let $\Delta=L \cap \mathbb{R}_{+}^{n}$ be the standard simplex. Denote by $\mu$ the Lebesgue measure on $L$ induced from $\mathbb{R}^{n}$. Then

$$
\int_{\Delta} \exp \{\langle c, x\rangle\} d \mu=\sqrt{n} \sum_{j=1}^{n} \exp \left\{c_{j}\right\} \prod_{k=1, k \neq j}^{n}\left(c_{j}-c_{k}\right)^{-1}
$$

for all $c \in \mathbb{R}^{n}$ with pairwise distinct coordinates.

Proof. It is enough to prove this identity only for $c$ in Int $\mathbb{R}_{-}^{n}$, since by adding a suitable multiple of $(1, \ldots, 1)$ we can always put $c$ in the negative orthant. Let us introduce the function

$$
\psi(x)= \begin{cases}\exp \{\langle c, x\rangle\} & \text { if } x \in \mathbb{R}_{+}^{n} \\ 0 & \text { otherwise }\end{cases}
$$

The desired integral has the form $\int_{\mathbb{R}^{n}} \psi d \mu$, where $\mu$ is considered as a measure in $\mathbb{R}^{n}$ concentrated on $L$. If we apply the Fourier transform ${ }^{\wedge}: \varphi(x) \mapsto \hat{\varphi}(y)=$ $\int_{\mathbb{R}^{n}} \varphi(x) \exp \{-2 \pi i\langle y, x\rangle\} d x$, we obtain

$$
\int_{\mathbb{R}^{n}} \psi d \mu=\int_{\mathbb{R}^{n}} \hat{\psi} \overline{d \hat{\mu}}
$$

Here $\hat{\psi}(x)=\prod_{j=1}^{n}\left(2 \pi i x_{j}-c_{j}\right)^{-1}$, and $\mu$ is the measure on the orthogonal complement $L^{\perp}$ with the density $\exp \{-2 \pi i\langle a, x\rangle\}$ where $a=(1 / n, \ldots, 1 / n)$.

So the desired integral is equal to

$$
\sqrt{n} \int_{\mathbb{R}} \exp \{2 \pi i y\} \prod_{j=1}^{n}\left(2 \pi i y-c_{j}\right)^{-1}
$$

If $c$ has pairwise distinct coordinates, then the function has only simple poles $y_{j}=(1 / 2 \pi i) c_{j}$ with the residues $\left(\exp \left\{c_{j}\right\} / 2 \pi i\right) \prod_{k=1, k \neq 1}^{n}\left(c_{j}-c_{k}\right)$. Since $c \in$ Int $\mathbb{R}_{-}^{n}$, all $y_{j}$ belong to the upper half-plane. Choosing the contour consisting of the upper semicircle $R(\cos \varphi+i \sin \varphi), 0 \leq \varphi \leq \pi$, and of the interval $[-R, R]$, where $R \rightarrow+\infty$, by the Residue Theorem we obtain the desired value.

This formula was obtained in [11]. Podkorytov uses [11] induction on $n$ instead of the Fourier transform.

Now we can describe the general form of the integrals $\int_{K} \exp \{\langle c, x\rangle\} d x$, where $K$ is a cone.

(2.4) Proposition. Let $K$ be a polytopal convex $n$-dimensional cone in $\mathbb{R}^{n}$ without straight lines and let $K^{*}=\left\{x \in \mathbb{R}^{n}:\langle x, y\rangle \leq 0\right.$ for all $\left.y \in K\right\}$ be the polar of $K$. Then 
for all $c \in \operatorname{Int} K^{*}$ the integral

$$
\int_{K} \exp \{\langle c, x\rangle\} d x
$$

exists, and determines a function $s(K ; c)$ which is rational in $c \in \mathbb{C}^{n}$. Let $u_{1}, \ldots, u_{r}$ be unit vectors on the extreme rays of $K$. Then the set of singular points of $s(K ; c)$ is the union of hyperplanes $H=\bigcup_{j=1}^{r} H_{j}$, where $H_{j}=\left\{c \in \mathbb{C}^{n}:\left\langle c, u_{j}\right\rangle=0\right\}$.

Proof. By Example 2.1 it follows that the statement is true when $K$ is a simple cone. The cone $K$ can be subdivided into the union of simple cones $K=\bigcup_{j=1}^{m} K_{j}$ so that Int $K_{i} \cap$ Int $K_{j}=\varnothing$ when $i \neq j$, and the set of extreme rays of $K_{j}$, $j=1, \ldots, m$, coincides with the set of extreme rays of $K$ (see, for example, Lemma 4.6.1 of [12]). It follows that

$$
s(K ; c)=\sum_{j=1}^{m} s\left(K_{j} ; c\right)
$$

and that each singular point of $s(K ; c)$ is contained in $H$. Since $s(K ; c) \rightarrow \infty$ when $c \rightarrow c_{0} \in H, c \in K^{*}$, we conclude that the set of singular points coincides with $H$.

If $K+v, v \in \mathbb{R}^{n}$, is a shift of the cone $K$, then the integral $\int_{K+v} \exp \{\langle c, x\rangle\} d x$ determines the meromorphic function $\exp \{\langle c, v\rangle\} s(K ; c)$. We denote it by $s(K+v ; c)$.

The following result provides a decomposition of an exponential integral over the whole polytope into a linear combination of the integrals over facets.

(2.5) Lemma. Let $P \subset \mathbb{R}^{n}$ be a convex $n$-dimensional polytope and let $\Gamma_{i}$, $i=1, \ldots, m$, be the set of its facets. Let $\mu_{i}$ be the Lebesgue measure on the affine hull of $\Gamma_{i}$. Denote by $\mathbf{n}_{i}$ the outer unit normal to $\Gamma_{i}$. Then

$$
\int_{P} \exp \{\langle c, x\rangle\} d x=\frac{1}{\langle\lambda, c\rangle} \sum_{i=1}^{m}\left\langle\lambda, n_{i}\right\rangle \int_{\Gamma_{i}} \exp \{\langle c, x\rangle\} d \mu_{i}
$$

for all $c \in \mathbb{C}^{n}, \lambda \in \mathbb{R}^{n}$, such that $\langle c, \lambda\rangle \neq 0$.

Proof. Without loss of generality we may assume that $\langle\lambda, \lambda\rangle=1$. Let us introduce the following differential form on $\mathbb{R}^{n}$ :

$$
\omega(x)=\exp \{\langle c, x\rangle\} u_{1} \wedge \cdots \wedge u_{n-1},
$$

where $\left\langle\lambda, u_{i}\right\rangle=0$ for all $i=1, \ldots, n-1$ and $\lambda \wedge u_{1} \wedge \cdots \wedge u_{n-1}=e_{1} \wedge \cdots \wedge e_{n}$. Here $e_{1}, \ldots, e_{n}$ is assumed to be the standard basis of $\mathbb{R}^{n}$. Note that $d \omega=$ $\exp \{\langle c, x\rangle\} e_{1} \wedge \cdots \wedge e_{n}$. Applying the Stokes formula to $P$ and $\omega$, we obtain the desired result. 
Now we are able to prove the main result of this section.

(2.6) Theorem. Let $P \subset \mathbb{R}^{n}$ be a convex $n$-dimensional polytope. For each vertex $v \in$ Vert $P$ of $P$, let us define the cone $K_{v}$ as the smallest convex cone with the vertex $v$ which contains $P$. Then

$$
\int_{P} \exp \{\langle c, x\rangle\} d x=\sum_{v \in \operatorname{Vert} P} s\left(K_{v} ; c\right)
$$

for all $c \in \mathbb{C}^{n}$ such that $c$ is a regular point of all functions $s\left(K_{v} ; c\right)$.

Proof. Let us choose $\lambda \in \mathbb{R}^{n}, \lambda \neq 0$, such that $\lambda$ is not orthogonal to any edge of $P$. Consecutively applying Lemma 2.5 to $P$, then to its facets, to faces of codimension 2 and so on, finally we obtain a decomposition

$$
\int_{P} \exp \{\langle c, x\rangle\} d x=\sum_{v \in \text { Vert } P} \exp \{\langle c, v\rangle\} q_{v}(c),
$$

where $q_{v}(c)$ are homogeneous rational functions, $q_{v}(t c)=t^{-n} q_{v}(c)$ for all $t \in \mathbb{C}$. Now we have to prove that in fact $q_{v}(c)=s\left(K_{v}-v ; c\right)$.

Let us choose $w \in$ Vert $P$. Consider an open set $U_{w} \in \mathbb{R}^{n}$ such that for all $c \in U_{w}$

$$
\langle c, w\rangle\rangle\langle c, v\rangle \quad \text { if } \quad v \in \operatorname{Vert} P \text { and } v \neq w
$$

and $U_{w}$ does not contain any singular point of $s\left(K_{v} ; c\right), v \in$ Vert $P$. Then we have

$$
\begin{aligned}
\lim _{t \rightarrow+\infty} & t^{n} \exp \{-t\langle c, w\rangle\} \int_{P} \exp \{t\langle c, x\rangle\} d x \\
= & \lim _{t \rightarrow+\infty} t^{n} \exp \{-t\langle c, w\rangle\} \int_{K_{w}} \exp \{t\langle c, x\rangle\} d x \\
= & \int_{K_{w}-w} \exp \{\langle c, x\rangle\} d x \quad \text { for all } c \in U_{w^{\prime}} .
\end{aligned}
$$

The first equality holds since the integrals outside an arbitrary small neighborhood of the vertex $w$ are asymptotically negligible when $t$ tends to infinity. The second equality holds since $s\left(K_{w}-w ; c\right)$ is a homogeneous function. Similarly, we have

$$
\lim _{t \rightarrow+\infty} t^{n} \exp \{-t\langle c, w\rangle\} \sum_{v \in \text { Vert } P} \exp \{t\langle c, v\rangle\} q_{v}(t c)=q_{w}(c)
$$

for all $c \in U_{w}$. So $q_{w}(c)$ and $s\left(K_{w} ; c\right)$ coincide on a certain open set in $\mathbb{R}^{n}$. Since these functions are rational they coincide everywhere. 
This theorem was first proved by Brion [3] in the case of a rational polytope $P$. This restriction arose because Brion obtained the result as a limit case of his theorem on integral points in convex integral polytopes (see Section 4). Later elementary proofs were given by A. G. Khovanskii and A. V. Puhlikov (1989) and independently by the author [2]. These proofs are essentially different, here we have given a modified version of the proof from [2]. Note that Theorem 2.6 follows from Example 2.3 and the observation that each polytope can be subdivided into simplices without new vertices. We gave here a different proof based on certain asymptotics since this method also appears to be useful in the case of discrete exponential sums.

\section{Computing the Volume of a Polytope}

We turn to the application of exponential integrals to computation of the volume of a convex polytope. It is known that this problem is \# $P$-complete if the polytope is given by its vertices or by its facets (see [8] and [4]). Nevertheless, there exist a probabilistic approximative algorithm which, for any given $\varepsilon>0$, computes the volume of a convex body given by its oracle with relative error less than $\varepsilon$, in a time which is polynomial in $\varepsilon^{-1}$ [5], and a deterministic polynomial-time algorithm which computes the volume of an $n$-dimensional convex body given by its oracle, with a relative error bounded by $n^{(3 / 2) n}$ (see [6]). The volume of an $n$-dimensional convex body given by its oracle with relative error less than (const $\cdot(n / \log n))^{n}$ cannot be computed in polynomial time (see [1]).

If we put $c=0$, then the Exponential Integral 1.1 transforms into the volume of $P$. However, the point $c=0$ is singular for all functions $s\left(K_{v} ; c\right)$. So to compute vol $P$ approximately, we have to calculate Integral 1.1 at a nonzero point $c$ which is close to 0 .

By arithmetic operations we mean addition, subtraction, division, multiplication, and comparison of real numbers. We begin with the computation of $s(K ; c)$.

(3.1) Lemma. Assume that a convex polytopal $n$-dimensional cone $K \subset \mathbb{R}^{n}$ without straight lines is given as convex hull of its extreme rays, so $K=\operatorname{co}\left\{u_{1}, \ldots, u_{m}\right\}$ for some given vectors $u_{1}, \ldots, u_{m} \in \mathbb{R}^{n}$. Then, for any given $c \in \mathbb{C}^{n}$, we can compute the value $s(K ; c)$ (or decide that $c$ is a singular point) using $O\left(n^{3} \cdot m \cdot\left(\begin{array}{l}m \\ n\end{array}\right)\right)$ arithmetic operations.

Proof. If $K$ is a simple cone we can use Example 2.1 to compute $s(K, c)$. In general, we have to reduce the problem to the case of simple cones. To do this, we use the so-called "regular" subdivision. We consecutively move the points $u_{i} \mapsto v_{i}=t_{i} \cdot u_{i}, t_{i}>1, i=1, \ldots, m$, so that the following condition of general position holds: if $\operatorname{dim} \operatorname{co}\left\{u_{i_{1}}, u_{i_{2}}, \ldots, u_{i_{n+1}}\right\}=n$, then the points $v_{i_{1}}, v_{i_{2}}, \ldots, v_{i_{n+1}}$ are affinely independent. To construct the points $v_{1}, \ldots, v_{m}$ we need $O\left(m \cdot n^{3} \cdot\left(\begin{array}{c}m \\ n\end{array}\right)\right)$ arithmetic operations. 
Put $P=\operatorname{conv}\left\{v_{1}, \ldots, v_{m}\right\}$. Now we have to check for each of $\left(\begin{array}{l}m \\ n\end{array}\right)$ collection of vertices $I=\left\{v_{i_{1}}, \ldots, v_{i_{n}}\right\}$ whether their convex hull is a facet of $P$, such that $K_{I}=\operatorname{co}\left\{u_{i_{1}}, \ldots, u_{i_{n}}\right\}$ is an $n$-dimensional convex cone. For a given collection this procedure requires $\mathrm{O}\left(n^{3} \cdot m\right)$ arithmetic operations. The set of such facets gives us a covering of $K$ by simple cones $K_{I}=\operatorname{co}\left\{u_{i_{1}}, \ldots, u_{i_{n}}\right\}$ such that each point in $K$, except points in a set of measure zero, belongs exactly to two such cones. (If we choose facets which are "visible" from the point 0 , then we obtain the regular subdivision mentioned above, but it is enough to use the constructed covering.) Now we have $s(k ; c)=\frac{1}{2} \sum_{I} s\left(K_{I} ; c\right)$.

Since we are interested in complexity theory, let us restrict ourselves to integral polytopes given by their vertices $v_{1}, \ldots, v_{m} \in \mathbb{Z}^{n}$. We use the notion of input size (see, for example, [6]). So for a given $v=\left(v_{1}, \ldots, v_{n}\right) \in \mathbb{Z}^{n}$, size $v \approx$ $n+\log \left(\left|v_{1}\right|+1\right)+\cdots+\log \left(\left|v_{n}\right|+1\right)$.

(3.2) Theorem. There exists an algorithm which, for any given integral vertices $v_{1}, \ldots, v_{m} \in \mathbb{Z}^{n}$ and any number $\varepsilon>0$, computes the volume of the polytope $P=$ $\operatorname{conv}\left\{v_{1}, \ldots, v_{m}\right\}$ with relative error less than $\varepsilon$ using a number of arithmetic operations which is polynomial in $\max \left\{\right.$ size $v_{1}, \ldots$, size $\left.v_{m}\right\}, \log \varepsilon$, and linear in

$$
\sum_{v \in \operatorname{Vert} P}\left(\begin{array}{c}
e(v) \\
n
\end{array}\right)
$$

where $e(v)$ denotes the number of edges containing $v$.

Proof. We have to choose sufficiently small $c \in \mathbb{R}^{n}$ and then compute Integral 1.1 using Theorem 2.6 and Lemma 3.1. First, let us determine the set of edges of $P$. For two given vertices $v_{i}, v_{j}$ the problem of finding whether $\left[v_{i}, v_{j}\right]$ is an edge of $P$ is a linear programming problem which can be solved in a time which is polynomial in $n, \max \left\{\operatorname{size} v_{1}, \ldots\right.$, size $\left.v_{m}\right\}$. This is the only case where size $v_{i}$ is involved. So we have determined the sets of extreme rays of $K_{v_{i}}$.

Now we have to construct a point $c$ which is separated from the sets of singular points of $s\left(K_{v_{i}} ; c\right)$ with some guarantee. Let $\left\{H_{j}=\left\{x:\left\langle x, u_{j}\right\rangle=0\right\}, j \in J\right\}$ be the set of all hyperplanes orthogonal to some edge of $P$. Put $c(t)=\left(1, t, t^{2}, \ldots, t^{n-1}\right) \in \mathbb{Z}^{n}$ for $t \in \mathbb{Z}$. Now $\left\{p_{j}(t)=\left\langle u_{j}, c(t)\right\rangle, j \in J\right\}$ is a family of nonzero polynomials. Using known bounds on the roots of a polynomial we can choose sufficiently large $t_{0}$ (but bounded by a polynomial in the input size) such that $\left|\left\langle c\left(t_{0}\right), u_{j}\right\rangle\right|>1$ for all $j \in J$. The complexity of this algorithm is linear in the number of hyperplanes.

Without loss of generality we may assume that $\varepsilon<\frac{1}{2}$. Put

$$
c=\frac{\varepsilon \cdot c\left(t_{0}\right)}{3 \cdot\left\|c\left(t_{0}\right)\right\| \cdot \max \left\{\left\|v_{1}\right\|, \ldots,\left\|v_{m}\right\|\right\}}
$$


Now we can compute $\int_{P} \exp \{\langle c, x\rangle\} d x$ using Theorem 2.6. Note that $s\left(K_{v_{i}} ; c\right)=\exp \left\{\left\langle c, v_{i}\right\rangle\right\} s\left(K_{v_{i}}-v_{i} ; c\right)$. To compute $s\left(K_{v_{i}}-v_{i} ; c\right)$ we can use Lemma 3.1. Since $\exp \left\{\left\langle c, v_{i}\right\rangle\right\}$ can be computed with error less than $\varepsilon_{0}$ using $O\left(\left(\log \varepsilon_{0}\right)^{2}\right)$ arithmetic operations, the proof follows.

If $e(v) \leq n+$ const for all $v \in \operatorname{Vert}(P)$, then the algorithm has a polynomial complexity. In the case when $e(v)=n$ for all $v \in \operatorname{Vert}(P)$, i.e., the polytope $P$ is simple, a polynomial algorithm of the computation of vol( $P)$ was presented in [9]. Lawrence used Gram's relation, which is in a sense a limit case of our Theorem 2.6 when $c=t \cdot c_{0}$ and $t \rightarrow 0$. A polynomial algorithm for computation of the volume of a simple polytope was also constructed in [13]. This algorithm is based on a certain decomposition of a simple polytope into the alternating sum of simplices.

Theorem 2.6 allows us to reduce computation of the volume to computation of $s\left(K_{v} ; c\right)$. Lemma 3.1 gives an estimation of the complexity of computation $s(K ; c)$ for a polytopal cone given as the convex hull of its extreme rays. In the following example we consider the dual situation, namely a cone is given as the intersection of half-spaces.

(3.3) Example. Let $K \subset \mathbb{R}^{n}$ be an $n$-dimensional convex polytopal cone without straight lines, which is given as the intersection $K=\bigcap_{j=1}^{m} H_{j}$ of $m$ half-spaces $H_{j}=\left\{x \in \mathbb{R}^{n},\left\langle a_{j}, x\right\rangle \geq 0\right\}$, where $a_{j} \in \mathbb{R}^{n}$. We shall prove that $s(K ; c)$ is a linear combination of not more than $\left(\begin{array}{l}m \\ n\end{array}\right)$ simplest rational functions of the type $\prod_{j=1}^{n}\left(\left\langle b_{j}, c\right\rangle\right)^{-1}$ for some $b_{j} \in \mathbb{R}^{n}, j=1, \ldots, n$. We shall show how to construct this decomposition.

Let us consider the inclusion $\varphi: K \rightarrow \mathbb{R}^{m}, \varphi(x)=\left(\left\langle a_{1}, x\right\rangle, \ldots,\left\langle a_{m}, x\right\rangle\right)$. Denote $L=\varphi\left(\mathbb{R}^{n}\right)$. Then we have $\varphi(K)=\mathbb{R}_{+}^{m} \cap L$. Let $\mu$ be the Lebesgue measure on $L$. For $c \in \mathbb{R}_{-}^{m}$ define $\psi(x)$ as in Example 2.3. Then we have

$$
s(K ; A c)=\operatorname{det}^{-1 / 2}\left(A A^{\natural}\right) \int_{\mathbb{R}^{m}} \psi d \mu
$$

where the matrix $A$ consists of the columns $a_{1}, \ldots, a_{m}$. Put $k=m-n$. Applying the Fourier transform, we finally obtain

$$
s(K ; A c)=\int_{\mathbf{R}^{k}} \prod_{j=1}^{m}\left(2 \pi i\left\langle a_{j}, y\right\rangle-c_{j}\right)^{-1} d y,
$$

where $c=\left(c_{1}, \ldots, c_{m}\right)$.

Let us consider the set $H$ of complex hyperplanes

$$
H_{j}=\left\{x \in \mathbb{C}^{k}: 2 \pi i\left\langle x, a_{j}\right\rangle-c_{j}=0\right\}, j=1, \ldots, m .
$$


Assume that $c \in \mathbb{R}^{n}$ is "generic," namely that there is no point in $\mathbb{C}^{k}$ which belongs to more than $k$ hyperplanes in the set $H$. For $J \subset\{1, \ldots, m\}$, card $J=k$, let us denote by $x_{J}$ the common point (if one exists) of the hyperplanes $H_{j}, j \in J$. Put

$$
R_{J}(c)=\left|\bigwedge_{j \in J} a_{j}\right|^{-1} \prod_{j \xi J}\left(2 \pi i\left\langle a_{j}, x_{J}\right\rangle-c_{j}\right)^{-1}
$$

Note that $R_{f}(c)$ is a rational function having the desired type. Using the decomposition $\mathbb{R}^{k}=\mathbb{R}^{k-1} \oplus \mathbb{R}$, we represent the integral (3.1) as an iterated one. We successively apply the Residue Theorem to the integral with $c$ chosen from a small neighborhood of the generic point $c_{0}$. Finally we obtain a linear combination containing $O\left(\left(\begin{array}{l}m \\ n\end{array}\right)\right)$ summands of the form $R_{J}(c)$. Since $s(K ; A c)$ is an analytic function in $c$, this decomposition holds for all $c \in \mathbb{C}^{n}$. The algorithm presented for the construction of this decomposition has a complexity which is polynomial in $n^{k}$.

There exists a decomposition of $K$ into a linear combination of $O\left(\left(\begin{array}{l}m \\ n\end{array}\right)\right)$ simple cones [14] (see also Section 6). Therefore our result also follows from the existence of this geometric decomposition. However, the author does not know how to construct the decomposition effectively.

By Example 3.3 it follows that for an $n$-dimensional polytope $P$, such that for all $v \in \operatorname{Vert}(P)$ the cone $K_{v}$ contains not more than $n+$ const facets, we can compute its volume in polynomial time.

Now we consider other methods of volume computation by means of exponential integrals.

(3.4) Laplace's Asymptotic Formula and the Volume of a Section. Assume that for a given polytope $P \subset \mathbb{R}^{n}$, we can compute $\int_{P} \exp \{\langle c, x\rangle\} d x$ for all $c \in \mathbb{R}^{n}$. Let $c \in \mathbb{R}^{n}$ be a vector such that $\langle c, x\rangle>0$ for all $x \in P$. Put

$$
P_{1}=P \cap\{x:\langle c, x\rangle=1\} .
$$

Then

$$
\text { vol } P_{1}=\lim _{m \rightarrow+\infty} \sqrt{\frac{m}{2 \pi}} \exp \{m\} \int_{P}\langle c, x\rangle^{m} \exp \{-m \cdot\langle c, x\rangle\} d x
$$

Proof. Let us denote $g(z)=\ln z-z, z>0, P_{z}=\{x \in P:\langle c, x\rangle=z\}$, and $\psi(z)=$ vol $P_{z}$. Then the integral is equal to $\int_{0}^{+\infty} \psi(z) \exp \{m \cdot g(z)\} d z$. Since $g(z)$ has the unique maximum $z=1$ and $g^{\prime \prime}(1)=-1$, then by Laplace's asymptotic formula we obtain the desired result.

An analogous formula holds for the intersection of a polytope with a space of larger codimension. 
To compute the integral we can use the formula

$$
\int_{P}\langle c, x\rangle^{m} \exp \{-m \cdot\langle c, x\rangle\} d x=\left.\frac{d^{m}}{d t^{m}} \int_{P} \exp \{t \cdot\langle c, x\rangle\} d x\right|_{r=-m}
$$

and then replace the differential operator $d^{m} / d t^{m}$ by the finite difference operator $(\Delta t)^{-m}(T-I)^{m}$, where $T: t \mapsto t+\Delta t$ is a shift operator and $I$ is the identity operator. So we can reduce the computation of the integral to the computation of $m+1$ exponential integrals over polytope $P$. Some examples and error estimations are given in [2]. In particular, we can compute approximately the volume of a section of the cube by a hyperplane.

(3.5) Decomposition into Facets and Stokes Formula. We can use Lemma 2.5 to obtain an expression of the exponential integral over $P$ as the sum of exponential integrals over facets. We can choose $\lambda \in \mathbb{R}^{n}$ orthogonal to some normal vector $\mathbf{n}_{i}$ in order to exclude "bad" facets from the sum. An example is given in [2].

\section{Exponential Sums}

We begin with three examples of exponential sums over integral polytopes and rational cones.

(4.1) Example (Simple Rational Cone). Let $K=\operatorname{co}\left\{u_{1}, \ldots, u_{m}\right\} \subset \mathbb{R}^{n}$ be a rational simple convex cone given as the conic hull of its extreme rays. So, $\operatorname{dim} K=m$ and $u_{1}, \ldots, u_{m} \in \mathbb{Z}^{n}$. Without loss of generality we assume that $u_{1}, \ldots, u_{m}$ are integral points on extreme rays of $K$ closest to 0 . Let us denote by $\Pi$ the following parallelepiped:

$\Pi=\left\{x \in \mathbb{R}^{n}: x=\alpha_{1} u_{1}+\alpha_{2} u_{2}+\cdots+\alpha_{m} u_{m}\right.$, where $0 \leq \alpha_{i}<1$ for all $\left.i=1, \ldots, m\right\}$.

Then we have (see, for example, Corollary 4.6 .8 of [12])

$$
\sum_{x \in \mathbb{Z}^{n} \cap K} \exp \{\langle c, x\rangle\}=\sum_{x \in \Pi \cap \cap \mathbb{Z}^{n}} \exp \{\langle c, x\rangle\} \cdot \prod_{i=1}^{m}\left(1-\exp \left\{\left\langle c, u_{i}\right\rangle\right\}\right)^{-1}
$$

for all $c \in \operatorname{Int} K^{*}$.

Let us denote by $L$ the integral lattice generated by $K \cap \mathbb{Z}^{n}$ and by $L_{0}$ the integral lattice generated by $u_{1}, \ldots, u_{m}$. Then card $\Pi \cap \mathbb{Z}^{n}$ is equal to the index $L: L_{0}$ of the subgroup $L_{0}$ in $L$.

(4.2) Example (Cube). Let $I^{n}=[0, m]^{n}$ be an $n$-dimensional cube. Then

$$
\sum_{x \in \mathbb{Z}^{n} \cap I^{n}} \exp \{\langle c, x\rangle\}=\prod_{i=1}^{n} \frac{\exp \left\{c_{i}(m+1)\right\}-1}{\exp \left\{c_{i}\right\}}
$$


for all $c=\left(c_{1}, \ldots, c_{n}\right)$. If $c_{i}=0$ for some $i$, then the corresponding factor is equal to $m+1$.

(4.3) Example (Regular Simplex). Let

$$
\Delta=\left\{x=\left(x_{1}, \ldots, x_{n}\right) \in \mathbb{R}_{+}^{n}, x_{1}+x_{2}+\cdots+x_{n}=m\right\},
$$

where $m \in \mathbb{N}$ is a regular simplex. Then it is easy to see that

$$
\sum_{x \in \Delta \cap \mathbb{Z}^{n}} \exp \{\langle c, x\rangle\}=h_{m}\left(C_{1}, \ldots, C_{n}\right)
$$

where $h_{m}$ is the full symmetric function of degree $m$ (see [10]), and $C_{i}=\exp \left\{c_{i}\right\}$.

Now we describe the general form of the function $\sum_{x \in \mathbb{K} \cap Z^{n}} \exp \{\langle c, x\rangle\}$ for a rational cone $K$.

(4.4) Proposition. Let $K$ be a rational polytopal convex cone in $\mathbb{R}^{n}$ without straight lines. Then for all $c \in \operatorname{Int} K^{*}$ the series

$$
\sum_{x \in K \cap \mathbb{Z}^{n}} \exp \{\langle c, x\rangle\}
$$

converges and determines a function $\sigma(K ; c)$ which is rational in $\mathrm{C}_{i}=\exp \left\{c_{i}\right\}$, $i=1, \ldots, n$. There exists a representation

$$
\sigma(K ; c)=\frac{P(c)}{\prod_{i=1}^{m}\left(1-\exp \left\{\left\langle c, u_{i}\right\rangle\right\}\right)},
$$

where $P(c)$ is a Laurent polynomial in $C_{i}$. If $K$ belongs to the half-space $x_{i} \geq 0$, then the degree of the numerator in $C_{i}$ does not exceed the degree of denominator. The set of singular points of $\sigma(K ; c)$ is the union of hyperplanes

$$
H_{j, k}=\left\{c \in \mathbb{C}^{n}:\left\langle c+2 \pi i k, u_{j}\right\rangle=0\right\}
$$

where $j=1, \ldots, m$ and $k \in \mathbb{Z}^{n}$.

Proof. By Example 4.1 it follows that the theorem is true when $K$ is a simple cone. Since $K$ can be subdivided into the union of simple rational cones $K=$ $\bigcup_{j=1}^{m} K_{j}$ it follows that the theorem is true in general (see Proposition 2.4).

If $K+v, v \in \mathbb{Z}^{n}$, is a shift of the cone $K$, then the series $\sum_{x \in K+v n \mathbb{Z}^{n}} \exp \{\langle c, x\rangle\}$ determines the rational in $C_{i}$ function $\exp \{\langle c, v\rangle\} \sigma(K ; c)$. We denote it by $\sigma(K+v ; c)$. Proposition 4.4 in different terms is known (see, for example Theorem 4.6.11 of [12]). We give its precise reformulation here in order to prove the following main result. 
(4.5) Theorem. Let $P=\operatorname{conv}\left\{v_{1}, \ldots, v_{m}\right\}$ be a convex integral polytope with the vertices $v_{1}, \ldots, v_{m} \in \mathbb{Z}^{n}$. For each $v \in \mathrm{Vert} P$, let us define the cone $K_{v}$ as the smallest convex cone with the vertex $v$ which contains $P$. Then

$$
\sum_{x \in P \cap \mathbb{Z}^{n}} \exp \{\langle c, x\rangle\}=\sum_{v \in \operatorname{Vert} P} \sigma\left(K_{v} ; c\right)
$$

for all $c \in \mathbb{C}^{n}$ such that $c$ is a regular point of all functions $\sigma\left(K_{v} ; c\right)$.

Proof. Let us consider an inclusion $\mathbb{R}^{n} \rightarrow \mathbb{R}^{n+1}, x \mapsto(x, 1)$. Let $\mathscr{K}=\operatorname{co}\{0, P\}$ be the conic hull of the polytope $P$. Putting $\hat{c}=(c, t)$ where $c \in \mathbb{R}^{n}$ and $t<0$, we obtain

$$
\sigma(\mathscr{K} ; \hat{c})=1+\sum_{m=1}^{+\infty} T^{m}\left(\sum_{x \in m P \cap \mathbb{Z}^{n}} \exp \{\langle c, x\rangle\}\right)
$$

where we denote $T=\exp \{t\}$. Using Proposition 4.4 we conclude that $\sigma(K ; \hat{c})$ is the rational generating function for the exponential sums over $m P$. The denominator of this function is equal to $\prod_{v \in \text { Vert } P}(1-T \exp \{\langle c, v\rangle\})$ and the degree of the numerator is not larger than the degree of the denominator. Then by Theorem 4.1.1. of [12] we conclude that for all $m \in \mathbb{N}$

$$
\sum_{x \in m P \cap \mathbb{Z}^{n}} \exp \{\langle c, x\rangle\}=\sum_{v \in \operatorname{Vert} P} \exp \{m\langle c, v\rangle\} Q_{v}(m ; c)
$$

where $Q_{v}(m ; c)=q_{v, n}(c) m^{n}+\cdots+q_{v, 0}(c)$ is a polynomial in $m$, whose coefficients are rational in $\exp \left\{c_{1}\right\}, \ldots, \exp \left\{c_{n}\right\}$.

Now we have to prove that actually $q_{v, i}(c) \equiv 0$ if $i>0$ and $q_{v, 0}=\sigma\left(K_{v}-v ; c\right)$. Then setting $m=1$ we obtain the desired result. We use the same ideas as in Theorem 2.6. Let us choose $w \in$ Vert $P$. Consider an open set $U_{w} \subset \mathbb{R}^{n}$ such that for all $c \in U_{w}$.

$$
\langle c, w\rangle>\langle c, v\rangle \quad \text { if } \quad v \in \operatorname{Vert} P \text { and } v \neq w
$$

and $U_{w}$ does not contain any singular point of $\sigma\left(K_{v} ; c\right), v \in$ Vert $P$. Then we have

$$
\begin{aligned}
& \lim _{m \rightarrow+\infty} \exp \{-m\langle c, w\rangle\} \sum_{x \in m P \cap \mathbb{Z}^{n}} \exp \{\langle c, x\rangle\} \\
& \quad= \lim _{m \rightarrow+\infty} \exp \{-m\langle c, w\rangle\} \sum_{x \in m K_{w} \cap \mathbb{Z}^{n}} \exp \{\langle c, x\rangle\} \\
& \quad=\sigma\left(K_{w}-w ; c\right) .
\end{aligned}
$$

The first equality holds since for an arbitrary small neighborhood $W$ of the vertex $w$ the sums outside the neighborhood $m W$ are asymptotically negligible when $m$ 
tends to infinity. The second equality is obvious. Applying the same construction to the right-hand side of decomposition (4.1), we conclude that the functions $q_{v, i}$ have the desired type.

This theorem was first proved by Brion [3] using the theory of toric varieties. Elementary proofs were given by A. G. Khovanskii and A. V. Puhlikov (1989) and later independently by the author. These proofs are different, here the author's proof is presented. Note that if $c=0$, then $\sum_{x \in m P \cap \mathbb{Z}^{n}} \exp \{\langle c, x\rangle\}$ transforms into the Ehrhart polynomial (see, for example, [12]). The point $c=0$ is singular for all functions $q_{v, 0}(c)$ and we can consider the Ehrhart polynomial as the value of $\sum_{v \in \operatorname{Vert} P} \sigma\left(K_{v} ; m c\right)$ at the point $c=0$. A result analogous to Theorem 4.5 holds for the integral points in the relative interior of $P$ (see [3]). It can be proved using similar arguments.

A. G. Khovanskii and A. V. Puhlikov also proved other remarkable identities between exponential sums and integrals.

\section{Counting Integral Points}

Now we turn to the application of exponential sums to the problem of counting the integral points in convex integral polytopes. This problem is more complicated than the problem of volume computation. It is \#P-complete even in the case of integral simplex. It is not known whether a polynomial algorithm exists for counting integral points even if the dimension is fixed. Unlike the case of exponential integrals it seems to be difficult to compute $\sigma(K ; c)$ even for simple cones.

(5.1) Definition. Let $K=\operatorname{co}\left\{u_{1}, \ldots, u_{m}\right\}$ be a simple cone. Assume that $u_{1}, \ldots, u_{m}$ are the integral points on extreme rays of $K$ closest to the origin. Let us denote by $I(K)$ the index of the sublattice $L_{0}$ generated by $u_{1}, \ldots, u_{m}$ in the lattice $L$ generated by $K \cap \mathbb{Z}^{n}$ (see Example 4.1).

It is known that $I(K)$ is equal to the g.c.d. of $m \times m$ minors of the matrix consisting of the columns $u_{1}, \ldots, u_{m}$ (see, for example, [12]).

(5.2) Lemma. Let $K=\operatorname{co}\left\{u_{1}, \ldots, u_{m}\right\} \subset \mathbb{R}^{n}$ be a simple cone given by its vectors $u_{1}, \ldots, u_{m} \in \mathbb{Z}^{n}$. Then for all $c \in \mathbb{C}^{n}$ we can compute the value of $\sigma(K ; c)$ (or decide that $c$ is a singular point) in a number of arithmetic operations which is polynomial in $n,\|c\|, \max \left\{\right.$ size $\left.u_{i}, i=1, \ldots, m\right\}$ and linear in $I(K)$.

Proof. By Example 4.1 we have

$$
\sigma(K ; c)=\sum_{x \in \Pi \cap \mathbb{Z}^{n}} \exp \{\langle c, x\rangle\} \cdot \prod_{i=1}^{m}\left(1-\exp \left\{\left\langle c, u_{i}\right\rangle\right\}\right)^{-1}
$$

So to compute $\sigma(K ; c)$ we have to find all integral points in the parallelepiped $\Pi$. We assume that $u_{1}, \ldots, u_{m}$ are closest to the origin integral points on extreme rays of $K$. As we have mentioned earlier (see Example 4.1) card $\Pi \cap \mathbb{Z}^{n}=$ 
$L: L_{0}=I(K)$. Note that it is enough to find a certain representatives of the cosets $L / L_{0}$, since if $y=\sum_{i=1}^{m} \beta_{i} u_{i}, \beta_{i} \in \mathbb{R}$, represents a coset in $L / L_{0}$, then the point $\hat{y}=\sum_{i=1}^{m}\left\{\beta_{i}\right\} u_{i} \in \Pi$ represents the same coset. We can construct in polynomial time a basis $e_{1}, \ldots, e_{m}$ of $L$ and a basis $d_{1}, \ldots, d_{m}$ of $L_{0}$ such that $d_{i}=\alpha_{i} e_{i}$ for all $i$ and for some $\alpha_{i} \in \mathbb{N}$ (see, for example, [7]). Then it is clear that vectors $\sum_{i=1}^{m} \beta_{i} e_{i}$, $0 \leq \beta_{i}<\alpha_{i}, \beta_{i} \in \mathbb{Z}$, are the desired representatives of $L / L_{0}$.

Now we are able to prove the main theorem of this section.

(5.3) Theorem. There exists an algorithm which, for any given integral vertices $v_{1}, \ldots, v_{m} \in \mathbb{Z}^{n}$ such that $P=\operatorname{conv}\left\{v_{1}, \ldots, v_{m}\right\}$ is a simple polytope, computes the number of integral points in $P$ using a number of arithmetic operations which is polynomial in $n, \max \left\{\right.$ size $v_{1}, \ldots$, size $\left.v_{m}\right\}$ and linear in $\sum_{v \in \mathrm{Vert} F} I\left(K_{v}-v\right)$.

Proof. The reasoning is essentially the same as in Theorem 3.1. We have to choose a sufficiently small $c$ which is a regular point for all functions $\sigma\left(K_{v} ; c\right)$ and then compute $\sum_{x \in P \cap Z^{n}} \exp \{\langle c, x\rangle\}$ using Theorem 4.2 and Lemma 5.2. It is enough to choose $c \in \mathbb{R}^{n}$ such that

$$
\|c\| \leq \frac{1}{3 \cdot\left(2 \cdot \max \left\{\left\|v_{1}\right\|, \ldots,\left\|v_{m}\right\|\right\}\right)^{n}}
$$

and then round the Exponential Sum 1.2 to the nearest integer.

Now we give an example of the computation of $\sigma(K ; c)$ for the cone $K$ given by its facets.

(5.4) Example. Let $a=\left(a_{1}, \ldots, a_{n}\right) \in \mathbb{Z}^{n}$. We assume that $a_{i}<0$ for some $i$. Put $K=\left\{x \in \mathbb{R}_{+}^{n}:\langle a, x\rangle=0\right\}$. Choose $c=\left(c_{1}, \ldots, c_{n}\right) \in$ Int $\mathbb{R}_{-}^{n}$ with the pairwise distinct coordinates. Let us consider the following series:

$$
F(z)=\sum_{x \in Z^{n}} \exp \{\langle c, x\rangle\} z^{\langle a, x\rangle}
$$

This series converges to $\prod_{j=1}^{n}\left[1 /\left(1-\exp \left\{c_{j}\right\} z^{a_{j}}\right)\right]$ for all $z$ in the circular annulus

$$
\max \left\{\exp \left\{\frac{-c_{j}}{a_{j}}\right\}, a_{j}<0\right\}<|z|<\min \left\{\exp \left\{\frac{-c_{j}}{a_{j}}\right\}, a_{j} \geq 0\right\} .
$$

By Cauchy's formula we have

$$
\sigma(K ; c)=\frac{1}{2 \pi i} \oint_{|z|=1} \frac{F(z)}{z} d z .
$$


Applying the Residue Theorem to this integral, we finally obtain

$$
\sigma(K ; c)=\sum_{j: a_{j}<0} \frac{\exp \left\{-c_{j}\right\}}{-a_{j}} \sum_{k=0}^{\left|a_{j}\right|-1} z_{j, k}^{-a_{j}} \prod_{m=1, m \neq j}^{n} \frac{1}{1-\exp \left\{c_{m}\right\} z_{j, k}^{a_{m}}},
$$

where we put $z_{j, k}=\exp \left\{\left(2 \pi i k-c_{j}\right) / a_{j}\right\}$.

Now it is clear that the complexity of the computation of $\sigma(K ; c)$ is linear in $\sum_{j: a_{j}<0}\left|a_{j}\right|$.

Exponential sums can also be applied to counting integral points in the intersection of a polytope with a hyperplane.

(5.5) Integral Points in Hyperplane Section. Let $P \subset \mathbb{R}^{n}$ be a convex polytope and let $a \in \mathbb{Z}^{n}$. Assume that $|\langle a, x\rangle|\left\langle L\right.$ for all $x \in P \cap \mathbb{Z}^{n}$ and for some $L \in \mathbb{N}$. Put $P_{1}=P \cap\left\{x \in \mathbb{R}^{n}:\langle a, x\rangle=0\right\}$. Then we have

$$
\operatorname{card} P \cap \mathbb{Z}^{n}=L^{-1} \sum_{k=0}^{L-1} \sum_{x \in P \cap \mathbb{Z}^{n}} \exp \left\{\frac{2 \pi i \cdot k\langle a, x\rangle}{L}\right\}
$$

Proof. For all $x \in P \cap \mathbb{Z}^{n}$ we have

$$
L^{-1} \sum_{k=0}^{L-1} \exp \left\{\frac{2 \pi i \cdot k\langle a, x\rangle}{L}\right\}= \begin{cases}1 & \text { if }\langle a, x\rangle=0 \\ 0 & \text { otherwise }\end{cases}
$$

Using Theorem 5.3 and Integral Points 5.5 we can define a class of polytopes for each of them the number of integral points inside can be easily computed. Using a similar approach we can also design algorithms for counting integral points which belong to the relative interior of an integral polytope.

\section{Integral Points in Nonrational Polytopes}

In this section we prove an analogue of Theorem 4.5 for nonrational polytopes.

Let $P \in \mathbb{R}^{n}$ be an $n$-dimensional convex polytope, let $K+v$ be an $n$-dimensional polytopal cone with the vertex $v$, and let $\rho: \mathbb{R}^{n} \rightarrow \mathbb{R}$ be a polynomial. Let us consider an exponential sum with polynomial density:

$$
\sum_{x \in P \cap \mathbb{Z}^{n}} \exp \{\langle c, x\rangle\} \rho(x)
$$

Together with this sum we can consider the series analogous to one which determines $\sigma(K+v ; c)$ :

$$
\sum_{x \in K+v \cap \mathbb{Z}^{n}} \exp \{\langle c, x\rangle\} \rho(x)
$$


Let us construct the following differential operator:

$$
D_{\rho}=\rho\left(\frac{\partial}{\partial c_{1}}, \ldots, \frac{\partial}{\partial c_{n}}\right) \text {. }
$$

For a polytopal $n$-dimensional cone $K+v$ with the vertex $v$ let us define

$$
s(K+v, \rho ; c)=D_{\rho} s(K+v ; c)
$$

It is clear that $s(K+v, \rho ; c)=\int_{K+w} \exp \{\langle c, x\rangle\} \rho(x) d x$ for all $c \in$ Int $K^{*}$. Applying the operator $D_{\rho}$ we obtain a $\rho$-analogue of Theorem 2.6:

$$
\int_{P} \exp \{\langle c, x\rangle\} \rho(x) d x=\sum_{v \in \operatorname{Vert} P} s\left(K_{v}, \rho ; c\right) .
$$

It turns out that for special polynomial densities $\rho$ we can prove a result analogous to Theorem 4.5 .

(6.1) Proposition. Assume that $\rho(x)=0$ for all $x \in \partial K+v$. Then for all $c \in \operatorname{Int} K^{*}$ the series (6.1) converges and determines a function $\sigma(K+v, \rho, c)$. This function has the representation

$$
\sigma(K+v, p ; c)=\sum_{k \in \mathbb{Z}^{n}} s(K+v, \rho ; c+2 \pi i k)
$$

The series (6.2) converges uniformly on any compact set which lies in

$$
\mathbb{C}^{n} \backslash \bigcup_{j \in J, k \in \mathbb{Z}^{n}}\left(H_{j}+2 \pi i k\right)
$$

where $H_{j}, j \in J$, is a certain finite set of hyperplanes in $\mathbb{C}^{n}$. In particular, $\sigma(K+v, \rho ; c)$ is an analytic function on $\mathbb{C}^{n} \backslash \bigcup_{j \in J, k \in \mathbb{Z}^{n}}\left(H_{j}+2 \pi i k\right)$.

Proof. First we prove the statement for a simple cone. Let $K=\operatorname{co}\left\{u_{1}, \ldots, u_{n}\right\}$. Let $u_{1}^{*}, \ldots, u_{n}^{*}$ be the dual basis, so $\left\langle u_{i}, u_{j}^{*}\right\rangle=\delta_{i, j}$. Set $\varphi(x)=\prod_{i=i}^{n}\left\langle u_{i}^{*}, x\right\rangle$. Then we have $\rho(x)=\varphi(x-v) \psi(x)$ where $\psi(x)$ is a certain polynomial. Let us consider the differential operator

$$
D_{\psi}=\psi\left(\frac{\partial}{\partial c_{1}}, \ldots, \frac{\partial}{\partial c_{n}}\right)
$$

Then the series (6.2) can be written as follows:

$$
\begin{aligned}
& \sum_{k \in Z^{n}} D_{\psi} s(K+v, \varphi(x-v) ; c+2 \pi i k) \\
& \quad=\left|u_{1} \wedge \cdots \wedge u_{n}\right| \sum_{k \in Z^{n}} D_{\psi} \exp \{\langle c+2 \pi i k, v\rangle\} \prod_{j=1}^{n} \frac{1}{\left(\left\langle-c-2 \pi i k, u_{j}\right\rangle\right)^{2}} .
\end{aligned}
$$


(Here we use the precise formula for the integral $\int_{K} \exp \{\langle c, x\rangle\} \varphi(x) d x$ which reduces to the integral $\int_{\mathbb{R}_{+}^{n}} \exp \{\langle c, x\rangle\} x_{1} \cdot x_{2} \cdots x_{n} d x$, see Example 2.1). When $\psi \equiv 1$ the series converges uniformly on each compact set in $\mathbb{C}^{n} \backslash \bigcup_{j \in J, k \in \mathbb{Z}^{n}}\left(H_{-}\right.$ $\left.{ }_{j}+2 \pi i k\right)$, where $H_{j}=\left\{x \in \mathbb{C}^{n}:\left\langle x, u_{j}\right\rangle=0\right\}$. If $\psi \not \equiv 1$, we can apply the known result on differentiating a convergent series of analytic functions. Then by Poisson summation formula we have that the sum of this series cannot differ from the sum (6.1) if $c \in \operatorname{Int} K^{*}$. So, for a simple cone $K$ we obtain

$$
\sigma(K+v, \rho ; c)=\left|u_{1} \wedge \cdots \wedge u_{n}\right| D_{\psi} \sum_{k \in \mathbb{Z}^{n}} \exp \{\langle c+2 \pi i k, v\rangle\} \prod_{j=1}^{n} \frac{1}{\left(\left\langle-c-2 \pi i k, u_{j}\right\rangle\right)^{2}} .
$$

Let us assume now that $K$ is not simple. Let $A_{j}, j \in J$, be the supporting hyperplanes of its facets. Let us choose an affine hyperplane $B$ in $\mathbb{R}^{n}$ such that $Q=K \cap B$ is a convex polytope and $B$ intersects with all affine subspaces of the form $A=A_{j_{1}} \cap A_{j_{2}} \cap \cdots \cap A_{j_{k}}$, dim $A>0$. By [14] it follows that there exists a representation

$$
Q=\sum_{\Delta \in \mathscr{D}} r_{\Delta} \Delta
$$

where $\Delta \in \mathscr{D}$ are simplices separated in $B$ by hyperplanes from the set $\left\{A_{j}\right\}$. Here $r_{\Delta}$ are integral numbers and the equality should be considered as an identity between characteristic functions $\chi_{Q}, \chi_{\Delta}$ which holds everywhere except on a set of Lebesgue measure zero in $B$. Put $K_{\Delta}=v+\operatorname{co}\{\Delta-v\}$. Then

$$
K=\sum_{\Delta \in \mathscr{D}} r_{\Delta} K_{\Delta}
$$

Since the desired statement is already proved for simple cones $K_{\Delta}$ the proof follows.

Now we can prove the main result of this section.

(6.2) Theorem. Let $P \subset \mathbb{R}^{n}$ be a convex $n$-dimensional convex polytope and let $\rho: \mathbb{R}^{n} \rightarrow \mathbb{R}$ be a polynomial such that $\rho(x)=0$ for all $x \in \partial P$. Then

$$
\sum_{x \in P \cap \mathbb{Z}^{n}} \exp \{\langle c, x\rangle\} \rho(x)=\sum_{v \in \text { Vert } P} \sigma\left(K_{v}, \rho ; c\right)
$$

for all $c \in \mathbb{C}^{n}$ which are regular points of the functions $\sigma\left(K_{v}, \rho ; c\right)$.

Proof. Let us consider the series

$$
\sum_{k \in \mathbb{Z}^{n}} \int_{P} \exp \{\langle c+2 \pi i k, x\rangle\} d x
$$


We have

$$
\int_{P} \exp \{\langle c+2 \pi i k, x\rangle\} \rho(x) d x=\sum_{v \in \mathrm{Vert} P} s\left(K_{v}, \rho ; c+2 \pi i k\right)
$$

By proposition 6.1 we conclude that the series (6.3) converges to

$$
\sum_{v \in \mathrm{Vert}} \sigma\left(K_{v}, \rho ; c\right)
$$

Furthermore, by the Poisson summation formula, we conclude that this sum cannot differ from

$$
\sum_{x \in P \cap \mathbb{Z}^{n}} \exp \{\langle c, x\rangle\} \rho(x)
$$

and the proof follows.

It seems that to compute $\sigma(K, \rho ; c)$ is much more difficult than to compute $\sigma(K ; c)$ for a rational cone $K$. One way to do this is to use $(6.2)$.

\section{Acknowledgments}

I am grateful to A. G. Khovanskii and to Imre Bárány for many helpful discussions.

\section{References}

1. I. Bárány, Z. Füredi, Computing the volume is difficult, Discrete Comput. Geom., 2(4) (1987), 319-326.

2. A. I. Barvinok, Calculation of exponential integrals (in Russian), Zap. Nauchn. Sem. LOMI, Teoriya Slozhnosti Vychislenii, 192(5) (1991), 149-163.

3. M. Brion, Points entiers dans les polyèdres convexes, Ann. Sci. Ėcole Norm. Sup (4), 21(4) (1988), 653-663.

4. M. Dyer, A. M. Frieze. On the complexity of computing the volume of a polyhedron, SIAM J. Comput., 17(5) (1988), 967-974.

5. M. Dyer, A. M. Frieze, R. Kannan, A random polynomial time algorithm for approximating the volume of convex bodies, J. Assoc. Comput. Mach., 38(1) (1991), 1-17.

6. M. Grötschel, L. Lovász, A. Schrijver, Geometric Algorithms and Combinatorial Optimization, Springer-Verlag, Berlin, 1988.

7. R. Kannan, A. Bachem, Polynomial time algorithms for computing the Smith and Hermite normal forms of an integer matrix, SIAM J. Comput., 8 (1979), 499-507.

8. L. G. Khachiyan, The problem of calculating the volume of a polyhedron is enumerably hard (in Russian), Uspekhi Mat. Nauk, 44(3) (1989), 179-180 (translated in Russian Math. Surveys, 44(3) (1989), 199-200).

9. J. Lawrence, Polytope volume computation, Math. Comp., 57(195) (1991), 259-271.

10. I. G. Macdonald, Symmetric Functions and Hall Polynomials, Clarendon Press, Oxford, 1979.

11. A. N. Podkorytov, Summation of multiple Fourier series over polyhedra (in Russian), Vestnik Leningrad. Univ. Mat. Mekh Astronom., (1) (1980), 51-58. 
12. R. P. Stanley, Enumerative Combinatorics, vol. 1, Wadsworth \& Brooks/Cole, Monterey, California, 1986.

13. A. N. Varchenko, Combinatorics and topology of the disposition of affine hyperplanes in real space (in Russian), Funktsional. Anal. i Prilozhen., 21(1) (1987), 11-22 (translated in Functional Anal. Appl., 21(1) (1987), 9-19).

14. A. N. Varchenko, I. M. Gel'fand, On Heaviside functions of a configuration of hyperplanes (in Russian), Funktsional. Anal. $i$ Prilozhen., 21(4) (1987), 1-18 (translated in Functional Anal. Appl., 21(4) (1987), 255-270).

Received December 1, 1991. 